\title{
A comparison of videolaryngoscopes for tracheal intubation in predicted difficult airway: a feasibility study
}

\author{
Maria Vargas ${ }^{1,2^{*}}$ (D) Antonio Pastore ${ }^{1}$, Fulvio Aloj ${ }^{2}$, John G. Laffey ${ }^{3}$ and Giuseppe Servillo 1,2
}

\begin{abstract}
Background: Videolaryngoscopy has become increasingly attractive for the routine management of the difficult airway. Glidescope ${ }^{\circledast}$ is well studied in the literature while imago V-Blade ${ }^{\circledast}$ is a recent videolaryngoscope. This is a feasibility study with 1:1 case-control sequential allocation comparing Imago V-Blade ${ }^{\circledR}$ and Glidescope ${ }^{\circledast}$ in predicted difficult airway settings.
\end{abstract}

Methods: Two senior anesthesiologists with no clinical experience in video assisted intubation but previously trained in a simulated scenario, performed the endotracheal intubations with Imago V-Blade ${ }^{\circledast}$ and Glidescope ${ }^{\circledast}$. A third experienced anesthesiologist supervised the procedures. Forty-two patients, 21 for each group, with the presence of predicted difficult airway according to the Italian guideline were included. The primary end point is the feasibility of intubation. The secondary end-points are the success to intubate in the first attempt, the intubation time, the Cormack and Lehane score view, the comparison of the intubation difficulty scale (IDS) score and the need for maneuvers to aid the endotracheal intubation comparing Imago V-Blade ${ }^{\oplus}$ and Glidescope ${ }^{\oplus}$.

Results: The intubation was achieved in 100\% of cases in both groups. No differences were found in the first-attempt success rate $(p=0.383)$, intubation time $(p=0.280)$, Cormack and Lehane score view $(p=0.799)$ and IDS score $(p=0.252)$. Statistical differences were found in external laryngeal pressure $(p=0.005)$, advancement of the blade $(p=0.024)$ and use of increasing lifting force $(p=0.048)$.

Conclusions: This feasibility study showed that the intubation with the newly introduced Imago V-Blade ${ }^{\circledast}$ is feasible. Further randomized and/or non-inferiority trials are needed to evaluate the benefit of Imago V-Blade ${ }^{\circledast}$ in this procedure.

Trial registration: Clinicaltrials.gov NCT02897518. Retrospectively registered 25 August 2016

Keywords: Videolaryngoscopes, Predicted difficult intubation, Intubation difficulty scale, Imago V-blade, Glidescope

\section{Background}

In recent years, videolaryngoscopy has become increasingly attractive for the routine management of the difficult airway. Videolaryngoscopes offer several advantages during endotracheal intubation. The Glidescope $e^{\bullet}$ is a videolaryngoscope for indirect laryngoscopy significantly different from Macintosh because of its rigid and $60^{\circ}$ angled blade. The

\footnotetext{
* Correspondence: vargas.maria82@gmail.com

${ }^{1}$ Section of Anesthesia and Intensive care, Department of Neurosciences, Reproductive and Odontostomatological Sciences, University of Naples "Federico II", Via Pansini 16, Naples, Italy

${ }^{2}$ Section of Anesthesia and Intensive care, Anesthesia and Intensive Care Unit, IRCCS Neuromed, Pozzilli, IS, Italy

Full list of author information is available at the end of the article
}

view of the glottis provided by the Glidescope ${ }^{\bullet}$ seems to be improved compared with the Macintosh laryngoscope in difficult airways [1]. During difficult intubations, the Glidescope $e^{\bullet}$ has been associated with more successful endotracheal intubation compared with the $\mathrm{C}^{-\mathrm{MAC}^{\circ}}$ videolaryngoscope [2].

The Imago V-Blade (Fig. 1) is a recent videolaryngoscope equipped with a wireless video-assisted stylet within it's $90^{\circ}$ angled blade. Both the Glidescope $e^{\circ}$ and Imago V-Blade ${ }^{\circ}$ have a digital camera at the tip of the blade extending the view angle beyond that of a standard Macintosh laryngoscope. The Imago V-Blade ${ }^{\circ}$ has a channel for the tracheal tube to be preloaded before the 


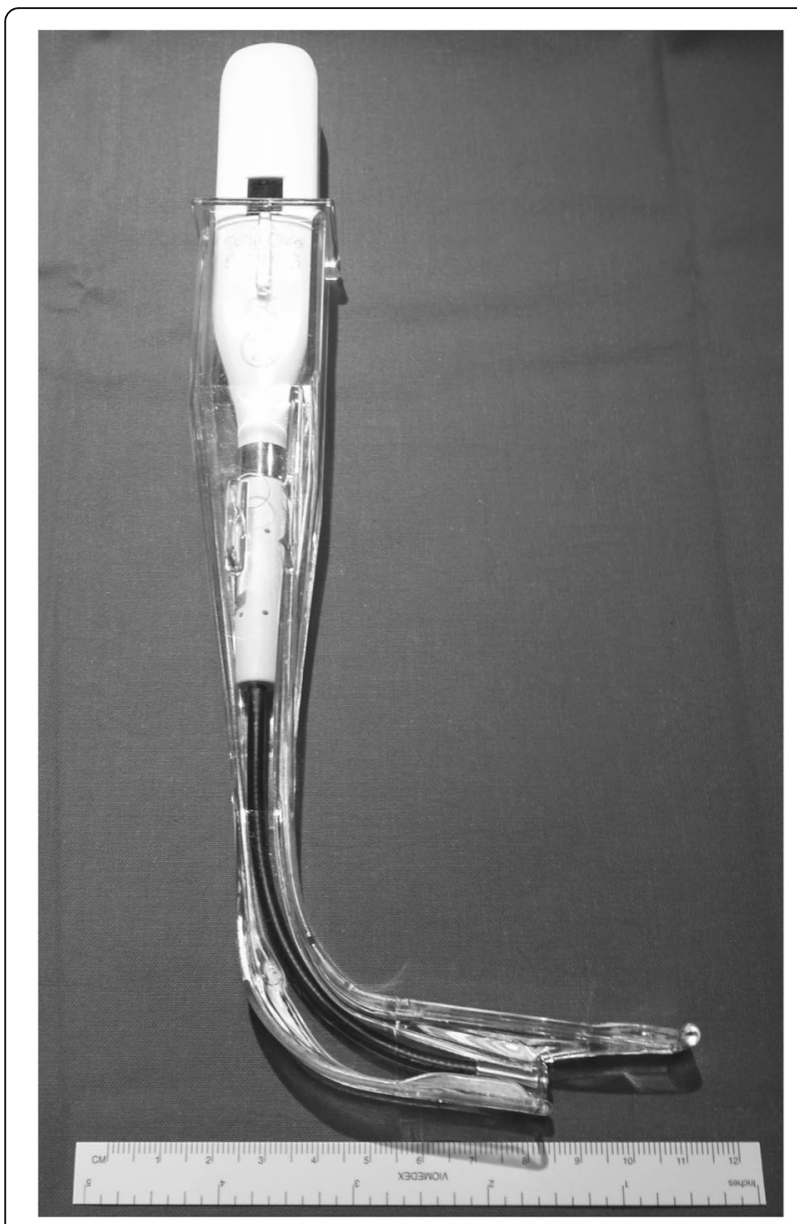

Fig. 1 Imago V-Blade ${ }^{\circledast} 90^{\circ}$ disposable blade with integrated channel for endotracheal tube. The shape of the blade is perpendicular to the main device axis

laryngoscopy. This is the first feasibility study comparing the use of the Imago V-Blade ${ }^{\circ}$ with the Glidescope ${ }^{\circ}$ in predicted difficult endotracheal intubation performed by non experienced anesthesiologist. The primary end point is the feasibility of intubation. The secondary end-points are the success to intubate in the first attempt, the intubation time, the Cormack and Lehane score view, the comparison of the intubation difficulty scale (IDS) score and the need for maneuvers to aid the endotracheal intubation comparing Imago V-Blade ${ }^{\bullet}$ and Glidescope ${ }^{\bullet}$.

\section{Methods}

\section{Study design and patient selection}

This is a feasibility study approved by the ethics committee of University of Naples "Federico II" (protocol number 123/15) and registered in clinical trial (Trial registration NCT02897518). All patients provided a written informed consent for study participation. Patients admitted to the operation rooms of University of Naples "Federico II" and requiring endotracheal intubation for general anesthesia were consecutively screened for the presence of predicted difficult airway according Italian guideline [3]. According to this guideline, the presence of one or more of the following parameters may be considered highly predictive of difficult intubation: Mallampati class 3-4, inter-incisor distance $<30 \mathrm{~mm}$, mental-thyroidal distance $<60 \mathrm{~mm}$, large prominence of superior incisors above inferior incisors uncorrectable with jaw-thrust, reduced head and neck motility, and reduced mental-jugular distance. Patients matching more than 1 of the previous criteria stated by the current Italian guideline were included in this case-controlled study. Patients were sequential allocated with a 1:1 ratio to each device/ group. The case group received endotracheal intubation with the Imago V-Blade ${ }^{\bullet}$ and the control group underwent tracheal intubation with the Glidescope ${ }^{\circ}$.

Patients 1) without criteria for predicted difficult airway; 2 ) those requiring emergency surgery; 3 ) aged $<18$ years; or 4) declined consent to participate, were excluded from this study.

During the reviewing process the primary end-point has been changed. The authors originally designed this study as non-inferiority study and then the primary end point was a comparison of the IDS score. However, since this is the first study evaluating a new device, we found more correct to call this study as feasibility study. As a consequence, the primary end-point is the feasibility of intubation with the new device Imago V-Blade ${ }^{\circ}$.

\section{End points}

The primary end point is the feasibility of intubation. The secondary end-points are the success to intubate in the first attempt, the intubation time, the Cormack and Lehane score view, the comparison of the intubation difficulty scale (IDS) score and the need for maneuvers to aid the endotracheal intubation comparing Imago VBlade ${ }^{\circ}$ and Glidescope ${ }^{\circ}$.

\section{Technical aspects}

Non-invasive blood pressure, electrocardiogram and pulse-oximetry were normally monitored for each patient. Patients were preoxygenated for $5 \mathrm{~min}$ with $100 \%$ oxygen. General anesthesia was induced with a standardized regimen that included intravenous fentanyl $(2 \mu \mathrm{g} / \mathrm{kg})$ and propofol $(2 \mathrm{mg} / \mathrm{kg})$. When the patient lost consciousness, bispectral index $<60$, rocuronium $(0.8 \mathrm{mg} / \mathrm{kg})$ was administered. A peripheral nerve stimulator (TOF-Watch ${ }^{\odot}$ Organon, Dublin, Ireland) was used to confirm that the train of- four ratio decreased to zero, which indicated an ideal intubation condition had been achieved. Mask ventilation with $100 \%$ oxygen was delivered to all patients during induction. 
All intubations were performed by two senior anesthesiologists (A and B) with 10 years of experience in conventional endotracheal intubation but without experience in video assisted intubation with Imago VBlade $^{\circ}$ or the Glidescope ${ }^{\circ}$. Anesthesiologist A performed all intubations with the Imago $\mathrm{V}$-Blade ${ }^{\circ}$ (group I) while anesthesiologist B used the Glidescope ${ }^{\circ}$ (group G). A third anesthesiologist $(C)$ with experience in video assisted intubation with both devices was present in the operation room. If the anesthesiologist A or B failed intubation after 2 attempts, anesthesiologist $C$ took over and completed the maneuver. Otherwise, after 3 attempts the patient was awakened and intubated via a fiberoptic bronchoscopy. Furthermore, the operating room was equipped by devices recommended by Italian guidelines for airway control and difficult airway management [3].

The successful intubation on the first attempt was defined as the tracheal tube placement with a single blade insertion. The successful intubation was confirmed by capnography and auscultation of lungs and stomach. The removal of the laryngoscope from the mouth and further manipulation of the laryngoscope inside the mouth also constituted an intubation failure. The intubation time was defined as the time period between the laryngoscopes passing the patient' $\mathrm{s}$ lips and the completion of a successful intubation. The Cormack and Lehane score [3] view was reported by both the laryngoscopists as the first own observation on the video screen just after the positioning of the videolaryngoscopes and without external tracheal maneuvers. The Intubation Difficulty Score (IDS) is a validated numerical description of the difficulty of intubation based on seven quantitative and qualitative aspects of the procedure, value 0 corresponding to ideal intubation conditions, values $1-5$ to slight difficulty, and values $>5$ to moderate to severe difficulty [4]. Maneuvers to aid the endotracheal intubations as readjusting patient's head, external laryngeal pressure, advancement or withdrawal the blade and increased lifting force, were collected by an independent data recorder observing the procedure.

\section{Description of device included in the study: Imago V- Blade $^{\circledR}$ and Glidescope ${ }^{\circledR}$}

The Imago V-Blade was equipped with a wireless video assisted stylet within the $90^{\circ}$ angled disposable blade. Endotracheal intubation with Imago V-Blade ${ }^{\circ}$ did not require a rigid stylet because it has a designed channel on the right for placement of the tracheal tube. This videolaryngoscope is inserted into the mouth in the midline, without displacing the tongue laterally, and advanced slowly until the epiglottis comes into view. The tip of the blade is then positioned in to the vallecula indirectly elevating the epiglottis for vocal cords exposure (Fig. 2). It is important to place the glottic opening in the centre of the monitor.

The Glidescope ${ }^{\circ}$ is a rigid video-laryngoscope with a $60^{\circ}$ angled blade connected by cable to a monitor. The tracheal tube used with the Glidescope ${ }^{\bullet}$ was pre-loaded with the manufacturer's pre-configured stylet because the Glidescope ${ }^{\circ}$ does not have a tracheal tube channel. The Glidescope ${ }^{\circ}$ is introduced into the middle of the oral cavity, without tongue displacement, gliding along the palate and the posterior pharynx until their tip is inserted into the vallecula or posterior to the epiglottis, if the epiglottis obscures the glottis.

Anesthesiologists A and B, with 10 years of experience in conventional endotracheal intubation but without experience in video assisted intubation, were given didactic instruction on the proper use of the Imago V-Blade ${ }^{\circ}$ and Glidescope ${ }^{\circ}$. As training, anesthesiologists $A$ and B each performed 60 intubations with the assigned videolaryngoscope in a manikin with three difficult airway scenarios: 20 intubations in a normal manikin without modifications, 20 intubations in a manikin with the tongue insufflated with $110 \mathrm{ml}$ of air, 20 intubations in a manikin with cervical immobilization. The anesthesiologist $\mathrm{C}$, with more than 100 clinical intubations with both devices, supervised the training.

\section{Statistical analysis}

Data are reported as means and standard deviations $( \pm$ $\mathrm{SD})$, proportions or median and range interquartiles

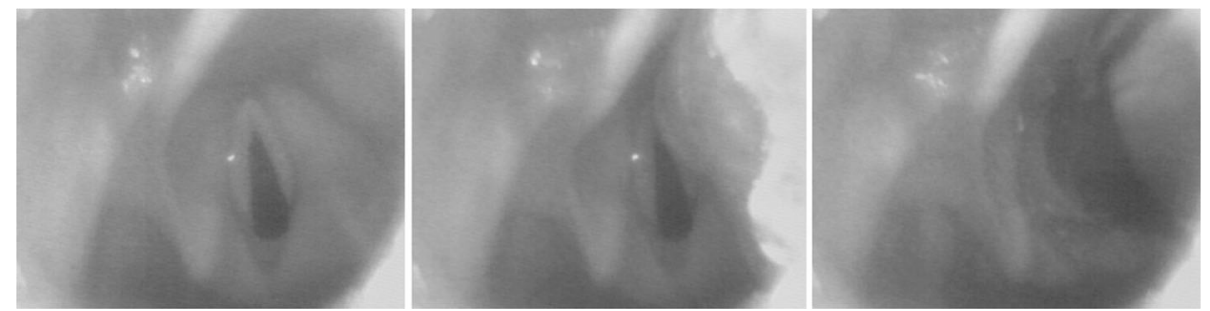

Fig. 2 Laryngeal view from the Imago V-Blade ${ }^{\varpi}$ used in this study. The left panel show the glottis view with the tip of the blade inserted into the vallecular. The middle panel shows the placement of the endotracheal tube in front of the vocal cords with the tip of the blade slightly elevating the epiglottis. The right panel shows the passage of the endotracheal tube though the vocal cords keeping the tip of the blade into the vallecula 
(IQR) as appropriate. Normal distribution was evaluated with the Shapiro-Wilk normality test. Comparisons between groups were performed with one-way ANOVA for continuous variables. Statistical significance (p) was set at 0.05 . Statistical analysis was obtained with SPSS (version 20.0, $\mathrm{IBM}^{\circ}$, USA). A statistical post-hoc power analysis on observed effect with probability level $(\alpha)$ set at 0.05 was performed to assess the power of this study. The sample size has been not calculated.

\section{Results}

Forty-two patients, 21 for each group, with the presence of predicted difficult airway according Italian guideline [3] were included in this study. Table 1 reported the main characteristics of included patients. The intubation was achieved in all patients $(21 / 21)$ in the group I and group G. The intubation success rate on the first attempt between Group I and Group G was similar (Fig. 3) $(p=0.383)$. In the group I, $1 / 21$ patient was intubated after the third attempt and 4/21 patients were intubated on the second attempt. In group G, $2 / 21$ patients were intubated on the second third attempt of endotracheal intubation. In the group I, the median time of endotracheal intubation was $23.10( \pm 5.56)$ seconds while in the group G $25.57( \pm 8.75)$ seconds without statistical significance $(p=0.280)$. The Cormack and Lehane scores view with the two different videolaryngoscopes were not different (Fig. 4) (C-L I/II/III/IV: group I - 6/5/9/1; group $\mathrm{G}-6 / 6 / 9 / 0 ; p=0.799)$.

Table 1 Main characteristics of included patients

\begin{tabular}{llll}
\hline & $\begin{array}{l}\text { Glidescope } \\
(21)\end{array}$ & $\begin{array}{l}\text { Imago } \\
(21)\end{array}$ & $p$ \\
\hline Age (mean \pm SD) & $62 \pm 10$ & $58 \pm 15$ & 0.636 \\
Gender (m/f) & $13 / 8$ & $10 / 11$ & 0.352 \\
BMI > 35 & $38.4 \pm 2.3$ & $38.6 \pm 1.52$ & 0.875 \\
Mallampati Class: & & & 0.635 \\
I & $1(4.8 \%)$ & 0 & \\
II & $5(23.8 \%)$ & $5(23.8 \%)$ & \\
III & $13(61.8 \%)$ & $12(57.1 \%)$ & \\
IV & $2(9.5 \%)$ & $4(19 \%)$ & \\
Previous difficult intubation & $2(9.5 \%)$ & $4(19 \%)$ & 1 \\
Inter-incisor gap $\leq 3$ cm & $6(28.6 \%)$ & $6(28.6 \%)$ & 1 \\
Thyromental distance $<6.5$ cm & $6(28.6 \%)$ & $6(28.6 \%)$ & 1 \\
Reduced jugular-mental distance & $5(23.8 \%)$ & $4(19 \%)$ & 1 \\
No possibility of maxillary prognatism & $3(14.3 \%)$ & $1(4,8 \%)$ & 1 \\
Head and neck movement <90 & $3(14.3 \%)$ & $4(19 \%)$ & 1 \\
Complete missing teeth & $4(19 \%)$ & $5(23.8 \%)$ & 1 \\
Macroglossia & $4(19 \%)$ & $3(14,3 \%)$ & 1 \\
Thyroid goiter & $4(19 \%)$ & $2(9.5 \%)$ & 1 \\
Tracheal deviation & $4(19 \%)$ & $5(23.8 \%)$ & 1 \\
\hline
\end{tabular}

The IDS score was less than 5 points in both groups (Fig. 5) (median and IQR for IDS: group G 1 (0-1), group I $1(0-2)(p=0.252)$.

Table 2 reports the maneuvers needed to aid intubation of included patients. There was no difference between devices in the need to readjust the patient's head. Glidescope $e^{\circ}$ required more external laryngeal pressure than Imago V-Blade ${ }^{\circ}$ (group G 13/21, group I 4/21, $p=$ $0.004)$. Advancement of the blade was more common in the group I than the group G (group I 11/21, group G 4/ 21, $p=0.024)$. The use of increasing lifting force more common in the group G (increasing lifting force: group G 10121, group I 4/21, $p=0.024)$.

\section{Discussion}

To our knowledge, this is the first feasibility study comparing Imago V-Blade ${ }^{\circ}$ and Glidescope $e^{\bullet}$ for patients with a predicted difficult airway. In this study we found that intubation was feasible with Imago V-Blade ${ }^{\circ}$ in all recruited patients. Furthermore, we found that Imago VBlade $^{\odot}$ and Glidescope ${ }^{\circ}: 1$ ) had a high intubation success rate on the first attempt, 2) a rapid time needed for endotracheal intubation, 3) Cormack and Lehane score view in most cases less than four and 4) needed different maneuvers to aid a successful endotracheal intubation. Imago $\mathrm{V}$-Blade ${ }^{\oplus}$ is a videolaryngoscope recently developed. This current prospective case-control study was designed to evaluate the performance of a new videolaryngoscopes, the Imago V-Blade ${ }^{\circ}$ and Glidescope ${ }^{\circ}$ since the Glidescope $^{\circ}$ is the most extensively evaluated and used videolaryngoscope.

According to the previous literature on videolaryngoscopes with $90^{\circ}$ blade, Imago V-Blade ${ }^{\circ}$ and Glidescope ${ }^{\circ}$ had a high success rate and a rapid time of intubation although the blades were differently angled $\left(90^{\circ}\right.$ vs $\left.60^{\circ}\right)$ [5-7]. Imago V-Blade ${ }^{\odot}$ has a $90^{\circ}$ blade with an integrated tube channel and the shape of the blade is perpendicular to the main device axis [8]. As similar devices, the light and view axis of Imago V-Blade ${ }^{\bullet}$ could be optically manipulated approximately $270^{\circ}$ [9]. Glidescope ${ }^{\circ}$ has a $60^{\circ}$ angled blade that does not require an alignment of the oral, pharyngeal and tracheal axes [10]. The light coming from the blade of the Glidescope ${ }^{\bullet}$ offers a visual axis of approximately $270^{\circ}$ to $300^{\circ}$ [9]. However, the intubation rate at first pass was reached after 5 patients in the Imago V-Blade ${ }^{\bullet}$ group and after 2 patients in the Glidescope $^{\bullet}$ group. Probably different device designs may result in different intubation success rate and then in a faster learning curve.

Surprisingly in this study we found $9 / 21$ patients in each group with grade 3 of Cormack and Lehane score. Despite these data suggesting a difficult endotracheal intubation, this one was successfully reached at first attempt in all but 6 patients. These are interesting data. 


\section{Learning curve}

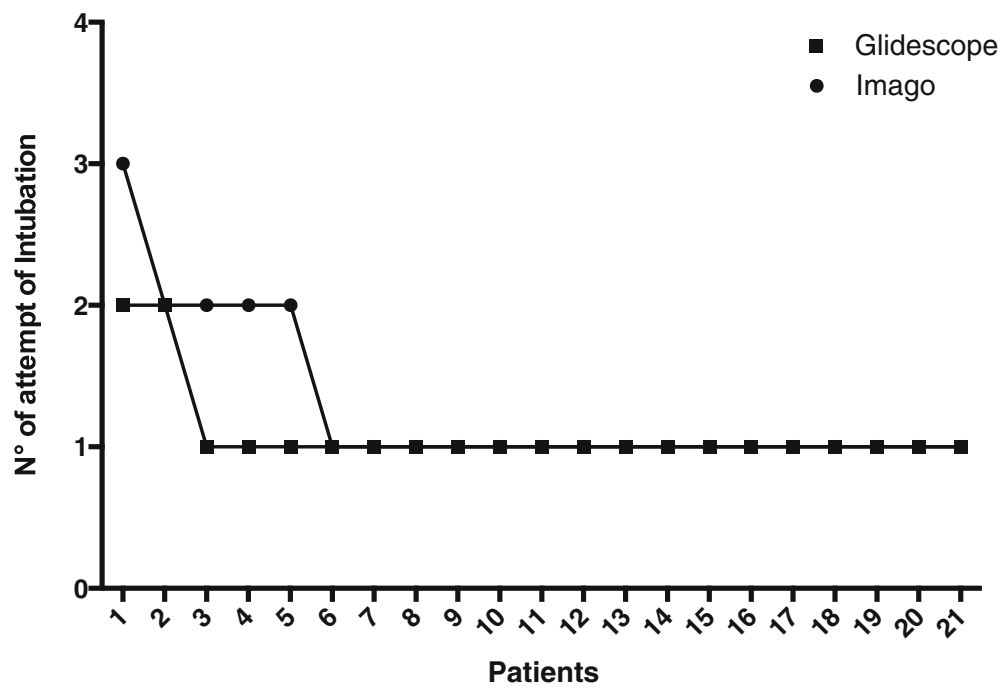

Fig. 3 Attempts of endotracheal intubation using the Imago V-Blade ${ }^{\oplus}$ and Glidescope ${ }^{\circledast}$ groups

Firstly, the Cormack and Lehane classification simply describe a view of the glottis and not the difficulty of the tube passage during the videolaryngoscopy [11]. Secondly, although a concerning grade of Cormack and Lehane score, in this study the IDS score showed an ideal condition of intubation in more than $70 \%$ of included patients. Probably the IDS score, as a descriptive method to assess difficult endotracheal intubation, may be more appropriate than Cormack and Lehane score to predict a difficult intubation during videolaryngoscopy [11]. Thirdly, the use of additional maneuvers to aid the intubation may facilitate the tube passage during videolaryngoscopy [8]. In line with current literature, Glidescope $^{\circ}$ needed more laryngeal pressure and increasing lifting force to successfully achieve endotracheal intubations $[8,12-14]$, while Imago V-Blade ${ }^{\circ}$ needed to be properly positioned at the center of the mouth. The

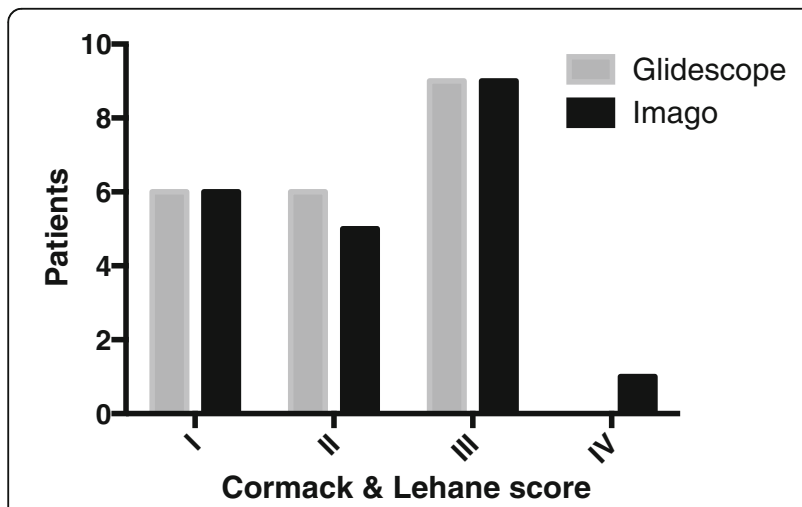

Fig. 4 Cormack and Lehane score view for patients included in the Imago V-Blade ${ }^{\circledast}$ (black bars) and Glidescope ${ }^{\circledast}$ (grey bars) groups technique for a successful endotracheal intubation whit Imago V-Blade ${ }^{\circ}$ is to position the camera at the center of the glottis with the tip of blade inserted into the vallecula indirectly elevating the epiglottis for laryngeal exposure.

\section{Limitations}

This study has some limitations. This is a feasibility study designed as prospective case-control study not as a randomized one. So, according to a post-hoc power analysis, this study is underpowered. The power analysis performed on observed effect size of successful intubation with 21 patients/group reached a power of 0.2 . We need 127 patients for each group to reach a power of

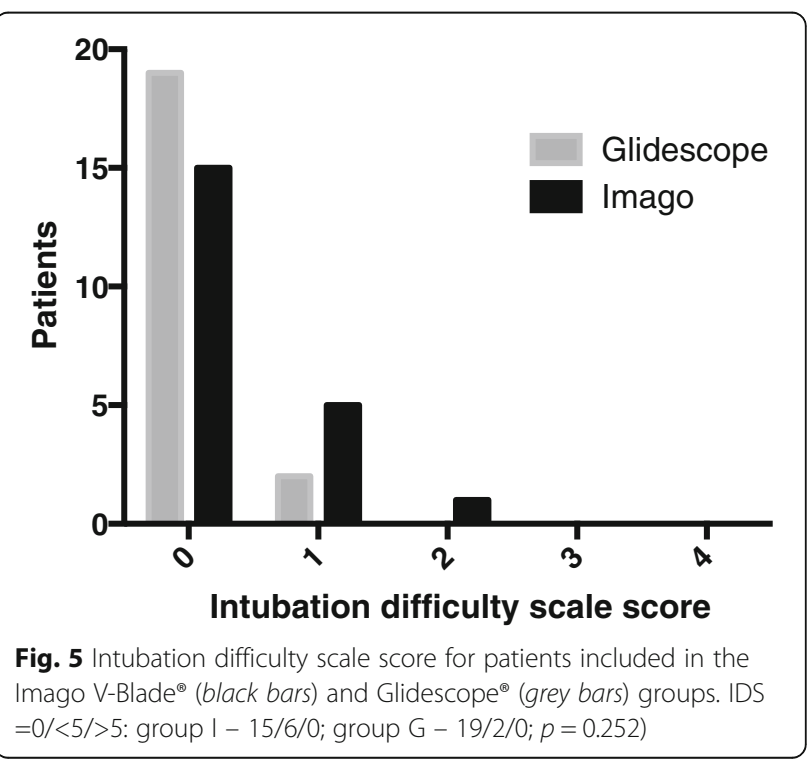


Table 2 Maneuvers to aid intubation in both groups

\begin{tabular}{llll}
\hline & $\begin{array}{l}\text { Glidescope } \\
(21)\end{array}$ & $\begin{array}{l}\text { Imago } \\
(21)\end{array}$ & $p$ \\
\hline Readjust patient's head & 0 & 0 & - \\
External laryngeal pressure & 13 & 4 & 0.004 \\
Advance blade & 4 & 11 & 0.024 \\
Withdraw blade & 8 & 4 & 0.179 \\
Increase lifting force & 11 & 4 & 0.024 \\
\hline
\end{tabular}

0.8. A randomized controlled trial may add further and complete information on this topic. In this study, two trained anesthesiologists performed the endotracheal intubation with the assigned device in order to evaluate the intubation success rate on the first attempt. The success of first intubation attempt may be affected by the experience of the operator [15]. Furthermore, Imago VBlade $^{\oplus}$ and Glidescope ${ }^{\oplus}$ have different angled blade that may slightly change the intubation maneuvers. According to these premises, we planned to not switch the anesthesiologists between the devices even if this point may introduce a bias.

\section{Conclusions}

This feasibility study showed that the intubation with the newly introduced Imago V-Blade ${ }^{\odot}$ is feasible. Further randomized and/or non-inferiority trials are needed to evaluate the benefit of Imago V-Blade ${ }^{\oplus}$ in this procedure.

\section{Abbreviations}

G: Glidescope ${ }^{\oplus}$; I: Imago V-Blade ${ }^{\oplus}$; IDS: Intubation difficulty scale

\section{Acknowledgements}

The authors would like to thank Professor Robert M Kacmarek and Professor Dario Bruzzese for their contributions in manuscript editing and reviewing.

\section{Funding}

The authors received no funding for this research.

\section{Availability of data and materials}

The authors would personally share the data with academic institutions after a reasoned request.

\section{Authors' contributions}

AP, FA, GS reviewed the literature, collected data, wrote the draft, revised the manuscript and approved the final version. MV and JGL reviewed the literature, collected data, performed statistical analysis, wrote the draft, revised the manuscript and approved the final version.

\section{Authors' information}

MV is a Member of the Task Force SIAARTI (Italian Society of Anesthesia and Intensive (are) Airway Management Study Group.

\section{Competing interests}

The authors declare that they have no competing interests.

\section{Ethics approval and consent to participate}

This prospective case-control study was approved by the ethics committee of University of Naples "Federico II" (protocol number 123/15). All patients provided a written informed consent for study participation.

\section{Author details}

1Section of Anesthesia and Intensive care, Department of Neurosciences, Reproductive and Odontostomatological Sciences, University of Naples "Federico II", Via Pansini 16, Naples, Italy. ${ }^{2}$ Section of Anesthesia and Intensive care, Anesthesia and Intensive Care Unit, IRCCS Neuromed, Pozzilli, IS, Italy. ${ }^{3}$ Section of Anesthesia and Intensive care, Department of Anesthesia, Critical IIIness and Injury Research Centre, Keenan Research Centre for Biomedical Science, St Michael's Hospital, University of Toronto, Toronto, Canada.

Received: 30 March 2016 Accepted: 10 February 2017

Published online: 20 February 2017

References

1. Nouruzi-Sedeh P, Schumann M, Groeben H. Laryngoscopy via Macintosh blade versus GlideScope: success rate and time for endotracheal intubation in untrained medical personnel. Anesthesiology. 2009;110:32-7.

2. Bruck S, Trautner H, Wolff A, Hain J, Mols G, Pakos P, Roewer N, Lange M. Comparison of the C-MAC ${ }^{\circledR}$ and Glidescope ${ }^{\oplus}$ videolaryngoscopes in patients with cervical spine disorders and immobilization. Anaestehsia. 2015;70:160-5.

3. SIAARTI task force for difficult airway. Recommendations for airway control and difficult airway management. Minerva Anestesiol. 2005;71:617-57.

4. Adnet F, Borron SW, Racine SX, Clemassy JL, Fournier JL, Plaisance P, Lapandry C. The intubation difficulty scale (IDS). Anesthesiology. 1997;87:1290-7.

5. Malik MA, Maharaj CH, Harte BH, Laffey JG. Comparison of Macintosh, Truview EVO2, Glidescope and Airwayscope laryngoscope use in patients with cervical spine immobilization. Br J Anaesth. 2008;101:723-30.

6. Liu EHC, Goy RWL, Tan BH, Asai T. Tracheal intubation with videolaryngosocpes in patients with cervical spine immobilization: a randomized trial of the Airway Scope ${ }^{\oplus}$ and the Glidescope ${ }^{\oplus}$. Br J Anesth. 2009;103:446-51.

7. Aziz MF, Abrons RO, Cattano D, Bayman EO, Swanson DE, Hagberg CA Todd MM, Brambrink AM. First-attempt intubation success of video laryngoscopy in patients with anticipated difficult direct laryngoscopy: a multicenter randomized controlled trial comparing the C-MAC D-blade versus the Glidescope in a mixed provider and diverse patient population. Anesth Analg. 2015. doi:10.1213/ANE.0000000000001084.

8. Corda DM, Riutort KT, Leone AJ, Qureshi MK, Heckman MG, Brull SJ. Effect of jaw thrust and cricoid pressure maneuvers on glottic visualization during glidescope videolaryngoscopy. J Anesth. 2012;26:362-8.

9. Levitan RM, Heitz JW, Sweeney M, Cooper RM. The complexities of tracheal intubation with direct laryngoscopy and alternative intubation devices. Ann Emerg Med. 2011;57:240-7.

10. Niforpoulou P, Pantazopoulos I, Demestha T, Koudouna E, Xanthos T. Videolaryngoscopes in the adult airway management: a topical review of the literature. Acta Anaesthsiol Scand. 2010;54:1050-61.

11. Healy DW, Maties O, Hovord D, Khepterpal S. A systematic review of the role of videolaryngoscopy in successful orotracheal intubation. BMC Anaesthesiol. 2012;12:32

12. Cooper RM, Pacey JA, Bishop MJ, McCluskey SA. Early clinicalexperience with a new videolaryngoscope (GlideScope) in 728 patients. Can J Anesth. 2005:52:191-8.

13. Kim JT, Na HS, Bae JY, Kim DW, Kim HS, Kim CS, Kim SD. Glidescope video laryngoscope: a randomized clinical trial in 203paediatric patients. $\mathrm{Br}$ Anaesth. 2008;101:531-4

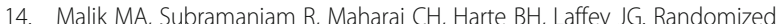
controlled trial of the Pentax AWS ${ }^{\circledast}$, Glidescope ${ }^{\circledast}$ and Macintosh laryngoscopes in predicted difficult intubation. Br J Anesth. 2009;103:761-8.

15. Griesdale DE, Liu D, McKinney J, Choi PT. Glidescope ${ }^{\oplus}$ videolaryngoscopy versus direct laryngoscopy for endotracheal intubation: a systematic review and meta-analysis. Can J Anesth. 2012;59:41-52. 